\title{
233. W. Manchot: Über die Verbindungen des Stickoxyds mit Ferro- und Cuprisalzen ${ }^{1}$ ).
}

[Mitteilung aus dem Chemischen Institut der Universität Würzburg.]

(Eingegangen am 7. Mai 1914.)

1. Darstellung krystallisierter Ferrustickoxyd-

Verbindungen.

Nachdem es mir unter Nitwirkung ron Huttner gelungen war, eine rote und eine braune Ferrostickoxyd-Verbindung im festen, krystallisierten Zustand zu erhalten, habe ich weitere Versucbe zur Gewinnung fester Ferrostickoxyd-Verbiudungeu angestellt. Hierbei knüpite ich unter Mitwirkung des Hrn. E. Merry an die von Mancbot und Huttner mitgeteilten, teilweise schon ron Péligot erwähnten Beobacbtungen au, daß beim Fällen der braunen Lösung von Ferrosulfat-Stickoxyd durcb eine Reihe ron Agenzien dunkle, stickoxydhaltige Niederschläge gefällt werden. Das Stickoxyd wird also nicht abgespalten, sondern geht mit dem Eisen in den Niederschlag hineiv. Als solche Fällungsagenzien kommen eine ganze Avzahl in Betracht, z. B. die Äzalkalien, Alkaliphosphate, Ferrocyankalium u. a. Es handelt sich also nur darum, geeiguete lösliche Salze von solchen Säuren oder eventuell solche lösliche Säuren selbst herauszugreifen, welche unlösliche Ferrostickoxyd-Verbindungen bilden. So einfach dies in Prinzip scheint, so werden die Versuche doch dadurch sehr erschwert, daß die ausfallenden Stickoxyd-Verbindungen meist unbeständiger Art sind, besonders aber dadurch, daß die wäßrigen, braunen Ferrostickoxyd-Lösungen, von denen man a!ısgebt, nicht ein ganzes Molekül Stickoxyd pro Atom Eisen gebundeu entbalten, sondern weniger, entsprechend dem Gleichgewicht $\mathrm{FeSO}_{4}+\mathrm{NO} \rightleftharpoons \mathrm{FeSO}_{4}, \mathrm{NO}$. Dieses Gleichgewicht liegt z. B. für eine Ferrosulfat-Lösung, welche 0.0429 Mole $\mathrm{FeSO}_{4}$ im Liter enthält, bei $0^{\circ}$ und $1 \mathrm{Atm}$. Partialdruck bei 17.8 I Stickoxyd pro Atom Eisen. Es sind also nur ruud $80 \%$ des vorbandenen Eisensulfats in der Lösung an Stickoxyd gebunden. Da nun die Ferrosalze durch die meisten derjenigen Fällungsagenzien, welche Ferrostickoxyd-Niederschläge fällen, für sich allein schon gefällt werden, so werden die dunklen Ferrostickoxyd-Niederschläge meist Beimengungen stickoxydireier Ferro.Niederschläge enthalten. Dieser Übelstand macht sich noch stärker benerkbar, wenn man, wie

ग) Frühere Mitteilungen A. 350, 368 [1906]; 372, 153 [1910]; 375, 30S [1910]; Z. Ang. 23, 2113 [1910]; 24, 13 [1911]; 25, 1055 [1912]; vergl. auch B. 45, 2869 [1912]; 46, 3514 [1913].

Berichte d. D. Chem. Gesellschaft. Jahrg XXXXVII. 
es für den präparativen Zweck notwendig ist, konzentriertere Ferrolösungen benutzt, weil mit zunehmender Eisen-Konzentration das Gleichgewicht nach links rückt, $d$. h. noch weniger Stickoxyd gebunden wird. Wendet man dagegen eine alkoholi sche Ferrolösung an, so läßt sich in der Aufangslösung die gesamte, 1 Mol. Stickoxyd pro Atom Eisen entsprechende Menge Stickoxyd an das Eisen bindeu. Hierbei entstebt allerdings eine neue Schwierigkeit dadurch, daß die Agenzien, mit welchen man nachher zu fällen hat, in Alkohol meist schwer oder nicht löslich sind.

Bei Ausführung der Versuche verfuhr man so, daß unter Benutzuug der früher beschriebenen Apparatur eine Lösung von Eisenchlorür in Alkohol bei $0^{n}$ mit Stickoxyd gesättigt und innerhalb der Stickoxyd-Atmosphäre mit einer, wenn möglicb, alkobolischen, sonst wäßrigen Lösung des Fällungsmittels vermischt wurde. Die Versuchsanorduung ermöglichte es, an einer etwaigen Vergrößerung des ahgesperrten Gasvolumens sofort zu erkenneu, ob beim Vermischen der Komponenten Stickoxyd abgespalten wird oder nicht.

Die brauchbarsten Resultate ergab die Untersuchung der Pho:sphat-Fällung. $1 \mathrm{~g}$ Eisencblorür wurde in $20 \mathrm{ccm}$ Alkohol bei $0^{\circ}$ mit Stickoxyd gesättigt und mit $1 \mathrm{Mol}$. Ammoniumphosphat, $\left(\mathrm{NH}_{4}\right)_{2} \mathrm{HPO}_{4}$ $(1.6 \mathrm{~g}$ in $25 \mathrm{ccm}$ Wasser), innerhalb der Stickoxyd-Atmosphäre vermischt.

Der tiefbraune Niederschlag wurde, nachdem das über der Flüssigkcit stehende Stickoxyd durch Wasserstoff verjagt war, filtriert, mit eiskaltem Sprit gewaschen und auf Ton rasch abgepreßt und in der früher beschriebenen Weise analysiert. Gef. $10.8 \mathrm{ccm}$ NO (reduziert) und $0.0309 \mathrm{~g}$ lie (Oxydul), entspr. $19.5 \mathrm{l} \mathrm{NO}$ auf $1 \mathrm{Fe}$. Bei cinem andren Versuch kamen anl $1 \mathrm{~g} \mathrm{FeCl}$ $50 \mathrm{cem}$ Sprit und $5 \mathrm{Mol}$. $\left(\mathrm{NH}_{4}\right)_{3} \mathrm{HPO}_{4}(8 \mathrm{~g}$ in $50 \mathrm{cen}$ Wasser) zur Anwendung.

Das obne Volumenänderung des abgesperrten Stickoxyds ausfallende, schwarzhraune, dicke Öl erstarrte beim Abkühlen mit EisKochsalzmischuug $\left(-15^{\circ}\right)$ rasch zu einer schwarzbraunen, krystallinischen Masse. Nach 1-stündigem Stehen wurde das über der Flüssigkeit stehende Stickoxyd durch Wasserstoff verjagt, die Flüssigkeit abgegossen und zweimal durch Sprit ersetzt, welcher auf $-15^{\circ}$ abgeküblt war. Das mit dem Glasspatel berausgenommene Produkt wurde auf einem Tonteller abgepreßt und analysiert.

Gef. $20.6 \mathrm{ccm}$ NO (reduziert) und $0.0516 \mathrm{~g} \mathrm{Fe}(0 x y d u l)$, ontspr. $22.3 \mathrm{l}$ anf $1 \mathrm{Fe}$ - $-30.25 \mathrm{~cm}$ NO (red.) und $0.0785 \mathrm{~g} \mathrm{Fe}(0 \mathrm{xydul}$ ), entspr. $21.5 \mathrm{l}$ auf 1 Fe. - $15.0 \mathrm{ccu}$ NO (red.) und $0.0474 \mathrm{~g} \mathrm{~F}$ e (Oxydul), entspr. 17.71 auf $1 \mathrm{Fe}$.

Der Niederschlag enthält Phosphorsäure. Konzentrierte Schwefelsäure färbt er tiefrot. Beim Stehen an der Luft oder in Wasser suspendiert, oxydiert er sich nicht sehr rasch, selbst wenn man mit 
Sauerstoff schüttelt. Bei dieser Oxydation wird er aber scbließlic ganz weiß.

Infolge dieses Farbenumschlags bei der Oxydation ist dieses Phosphat eine für diesen Typus von Stickoxyd-Verbindungen besonders charakteristische Substanz, denn Ferrophosphat ist weiß und auch Ferriphosphat fast farblos. Man bekommt daher in diesem Falle, wenn man von einer Ferrophosphat-Fällung ausgeht, aus weißem Ferrophosphat ein dunkles Ferrostickoxyd-phosphat, welches dana bei der Oxydation mit Sauerstoff fast farblos wird.

Daß auch fertig bereitetes, d. b. vorher gefälltes Ferrophosphat Stickoxyd aufnimmt, läßt sich hier, eben wegen dieser günstigen Farbeuverschiedenheiten, leicht erkennen. Insbesondere läßt es sich eben desbalb besonders gut erkenneo, da $B$ Ferrophosphat wirklich Stickoxyd bindet, und da $\beta$ es nicht etwa vom Stichoxyd nur oxydiert wird, weil sonst nicht ein dunkles, sondern ein helles Produkt entstehen müßte.

$1 \mathrm{~g}$ Ferrosulfat wurde in $20 \mathrm{ccm}$ Wasser gelöst und mit $2 \mathrm{Mol}$. Ammoniumphosphat in $20 \mathrm{ccm}$ Wasser innerhalb einer Wasserstoffatmosphäre gefällt. Darauf wurde, ohne die Flüssigkeit mit dem Niederschlag zu bewegen, der Wasserstoff durch Stickoxyd ersetzt, der Stand der Sperrflüssigkeit an der Bürette abgelesen und kräftig geschüttelt. Es fand eine schnclle Absorption des Stickoxyds statt und die weiße Fällung wurde tiefbraun. Sie erwies sich bei der qualitativen Untersuchung als Ferrostickoxyd-Verbindung und wurde an der Luft allmählich gelblichweiß. Der Versuch wurde auch quantitativ wiederholt und gefunden, daß von $0.5498 \mathrm{~g}$ Eisenvitriol von $19.92 \%$ Ferrogehalt $38.4 \mathrm{ccm}$ NO (red.) absorbiert wurden, entspr. $19.91 \mathrm{I}$ auf $1 \mathrm{Fe}$, also nahezu 1 Molekïl No, wobei der Feblbetrag wohl hauptsăchlich daher stammt, daß bei der in diesem Fall benutzten Versuchsanordnung die Anfangseinstellung der Bürette unvermeidlicherweise nicht ganz genau war.

Endlich wurde noch durch quantitative Versuche festgestellt, daß bei dem Fällen mit Ammoniumphosphat keine Abspaltung von Stickoxyd eintritt, z. B. $0.2262 \mathrm{~g}$ Eisenchlorür in $50 \mathrm{ccm}$ absolutem Alkobol absorbierten bei $0^{0} 25.5 \mathrm{ccm}$ NO (red.), entspr. $22.3 \mathrm{l}$ auf $1 \mathrm{Fe}$. Hierzu wurde genau $1 \mathrm{Mol}$. Ammoniumphosphat gelöst in $10 \mathrm{ccm}$ Wasser gegeben. Es war keinerlei Volumenänderung zu beobachten.

Aus diesen Versuchen geht mit Sicherheit hervor, daß die braune Fällung Ferrostickoxyd-phosphat, (FeNO) $\mathrm{HPO}_{4}$, ist.

Bei einer späteren, unter dankenswerter Mitwirkung meines Assistenten Dr. J. Haas ausgeführten Wiederbolung dieser Versucbe wurde gefunden, daß die Substanz durchaus nicht so zersetzlich ist, wie es zuerst den Anschein hatte. Vielmehr wird die Unhandlichkeit 
des Ferrostickoxyd-phosphats hauptsächlich durch den niedrigen Schmelzpunkt verursacht.

Es wurde deshalb bei der Darstellung noch niedrigere Temperatur in Anwendung gebracht und in folgender Weise verfahren: $1 \mathrm{~g}$ Eisencblorür in $50 \mathrm{ccm}$ Sprit bei 00 mit Stickoxyd gesättigt, wurde innerhalb der StickoxydAtmosphäre mit $8 \mathrm{~g} \mathrm{Ammoniumphosphat} \mathrm{in} 50 \mathrm{ccm}$ Wasser versetzt. Ein Clberschuß von Ammoniumphosphat hat sich als vorteilhaft erwiesen, es sebeint dabei aussalzend zu wirken. Das ausgefallene dunkle Ol wurde bei einstündigem Stehen in Eis-Kochsalz-Mischung fest. Nach dem Verjagen des Stickoxyds durch Wasserstoff wurde die überstehende Flüssigkeit abgegossen und zweimal durch Sprit, der mit Koblensăure-Aceton-Mischung abgekühlt war, ersetzt.

Die herausgenommene, braunschwarze Masse erweist sich bei der mikroskopischen Betrachtung als aus braunen, plattigen Krystallen bestehend, was übrigens auch schon bei den früberen Versuchen beobachtet wurde, nur erlanbte die stärkere Vorküblung eine bequemere Betrachtung, weil bei einigem Verweilen in Zimmertemperatur die Krystalle zu einem braunen (̈) schmelzen. Der Schmelzpunkt läßt sich im Röbrchen am Thermometer gut erkeuneu, er ist ziemlich scharf bei $+16^{\circ}$.

Ein andrer Teil dieser Substanz wurde mittels stark gekühlter Tonplatten abgepreßt und analysiert. Gef. $0.0463 \mathrm{~g} \mathrm{Fe}$ (Oxydul) und $17.85 \mathrm{ccm}$ NO (red.) entspr. 21.51 NO auf 1 Fe.

Damit ist zum ersten Mal eine Ferrostickoxyd-Verbindungderbraunen Reihe in festem, krystallisiertem Zustand erhalten, welche ein ganzes Molekül Stickoxyd auf ein Atom Eisen enthält. Die von Manchot und Huttoer') früher beschriebene feste Substanz ist eine Doppelverbindung von Ferrosulfat mit Ferrostickoxyd-sulfat, welche unter Abspaltung der Hälfte des gebundenen Stickoxyds entsteht und daher $1 / 2 \mathrm{NO}$ auf $1 \mathrm{Fe}$ enthălt. Nur in der roten Reihe ist ebenfalls von Mauchot und Huttner eine krystallisierte Verbindung mit dem Verbältnis 1 NO auf $1 \mathrm{Fe}$ bereits dargestellt worden.

Die Untersuchung verschiedener andrer Ferrostickoxyd-Niederschläge ergab ein im ganzen ähnliches Bild wie beim Phosphat, doch haben die Produkte sich bisher weniger gut charakterisieren lassen. Nachdem aber jetzt die Schwierigkeit erkannt ist, welche durch den niedrigen Erstarrungspunkt mancher dieser Substanzen entsteht, werden sich durch eine ausgiebige Anwendung sehr niedriger Temperaturen wohl auch die Fällungen mit Ferrocyankalium, Borax und namentlich mit Natriumarsenat, welche der Pbosphat-Fällung sehr äbnlich ist, noch charakterisieren lassen.

3) A. 872, 165 [1910]. 
Bei der Fällung mit Ätzkali, Ammoniak und Natriumcarbonat entsteht eine Schwierigkeit dadurch, daß die Ferrostickoxyd-Fällung in ibrer Farbe von Eisenhydroxyd zu wenig versehieden ist und gerade hier bei den zum Isolieren und Analysieren der Niederschläge unvermeidlichen Operationen sehr rasch Oxydation, sei es durch die Luft, sei es auf Kusten des gebundenen Stickoxyds selbst eintritt. Es gelang nur in einem Falle, bei der Zersetzung des mit Ätzkali gefäliten Niederschlages von Ferrostickoxyd-hydroxyd eive erbebliche Meoge Stickoxyd wieder abzuspalten, doch war die qualitative Stickoxyd-Reaktion auch sonst unzweifelbaft.

Evdlich wurde noch versucht, durch $Z$ usatz von Lösungen organischer Säuren, wie $\mathrm{O}$ xalsäure, Weinsäure, Milchsäure, Citronensäure und vieler andrer, schwer lösliche Ferrostickoxyd-Salze auszufällen, was aber in keinem Falle gelang. Von Interesse ist gleichwobl, daß es weder mit Oxalsäure selbst, noch mit Ammoniumoxalat gelang, ein scbwer lösliches Ferrostickoxyd-oxalat zu fällen unter Bedingungen, wo in Abwesenbeit von Stickoxyd sofort Ferrooxalat ausfiel. Es entspricht also dem so schwer löslichen, charakteristischen Ferrooxalat ein leicht lösliches Ferrostickoxydoxalat.

Die Ferrostickoxyd-Verbindungen sind also jetzt durch bestimmte und leicht reproduzierbare Eigenschaften und Unterscheidungsmerkmale sowohl gegenüber den Ferro- wie gegenüber den Ferrisalzen charakterisiert.

II. Einwirkuug von Bromwasserstoff auf das Gleichgewicht des Ferrostickoxyd-bromürs, FeBr, NO.

Wie ich fruiber gezeigt habe, wird die Menge des im Gleichgewicht von Ferrosulfat bezw. -chlorid, $\mathrm{FeSO}_{4}+\mathrm{NO} \rightleftharpoons \mathrm{FeSO}_{4}$, $\mathrm{NO}$, gebundenea Stickoxyds durch sukzessive verstärkten Zusatz von Sch wefelsäure bezw. Salzsäure zuerst herabgedrückt, steigt aber dann wieder an und erreicht schließlich den Grenzwert von 1 Molekül Stickoxyd auf 1 Atom Eisen. Neuerdings hat Hr. E. Merry auf meine Veranlassung die Wirkung von Bromwasserstoff untersucht.

\begin{tabular}{|c|c|c|c|c|c|c|}
\hline $\begin{array}{l}\text { Molek. } \mathrm{HBr} \\
\text { in } 1000 \mathrm{ccm}\end{array}$ & $\begin{array}{l}\text { Gebund. } \\
\text { auf } 1 \mathrm{Fe}(\end{array}$ & & Farbe & $\begin{array}{l}\text { Molek. } \mathrm{HBr} \\
\text { in } 1000 \mathrm{ccm}\end{array}$ & $\begin{array}{l}\text { Gebund. NO } \\
\text { auf } \backslash \mathrm{Fe} \text { (Lit.) }\end{array}$ & Farbe \\
\hline 0 & 17.45 & dun & rel grünbraur & an $\quad 9.35$ & 17.19 & rötlichbraun \\
\hline 1.918 & 10.46 & tief & rötlichbraun & 11.52 & 18.70 & vergl. unten \\
\hline 2.110 & 10.06 & > & $\gg$ & 12.20 & 19.60 & $" \quad \gg$ \\
\hline 3.836 & 12.41 & $\bowtie$ & - & 13.83 & 21.78 & " \\
\hline 4.54 & 12.65 & » & $D$ & 14.33 & 22.14 & » \\
\hline 6.40 & 13.55 & » & ఎ & 14.67 & 23.61 & \\
\hline
\end{tabular}


Bei allen Versuchen ist die Versucbstemperatur $0^{\circ}$, die Eisen-Konzentration $0.0127 \mathrm{Mol}$. Ferroammoniumsulfat in $1000 \mathrm{ccm}$. Diese Konzentration wurde nach den in der früheren Arbeit entwickelten Gesichtspunkten ausgewählt. Der Stickoxyddruck war bei allen Versuchen gleich dem Barometerstand, also praktisch überall gleich (1 Atm.). Die Farbe von ohne Bromwasserstoff bereiteten Ferrostickoxyd-Lösungen war unter sonst gleichen Bedingungen der Farbe der bromwasserstoff-haltigen sehr ählich, doch zeigten die bromwasserstoflfreien Lösungen einen deutlicheren Stich ins Grüne. Bei den letzten 5 Versuchen war die Farbe ein etwas schmutziges Schwarzbraun mit einem Stich ins Grünliche. Die Zahlen für Stickoxyd bedeuten bei Versuch Nr. 1 5-8 und 10 Mittelwerte aus je zwei nahe übereinstimmenden Ergebnissen, die übrigen entsprechen Einzelversuchen mit den unvermeidlichen Versuchsfehlern.

Die Ausführung der Versuche entspricht im wesentlichen der früheren Anordnung, nur wurde die folgende $A$ bänderung vorteilhait gefunden. Bei den Versuchen mit Salzsäure batte sich nämlich insofern eine experimentelle Schwierigkeit ergeben, als es für die Füllung des Apparates mit Stickoxyd notwendig ist, zunächst die Luft vollständig durch abwechselndes Evakuieren und Nachfüllen eines indifferenten Gases, z. B. Wasserstoff, zu rerdrängen und letzteres schließlich durch Stickoxyd za ersetzen. Befindet sich vun die konzentrierte Halugcnwasserstoffsäure bereits in dem evakuierten Raum, so wird bei den sehr konzentrierten Säuren etwas Halogenwasserstoff mitgerissen und dadurch die Konzentration des Halogenwasserstoffs in der Flūssigkeit niedriger werden. Üm dies zu vermeiden, gab man die konzentrierte Halogenwasserstoffsäure in einen auf das Absorptionsgefä $B$ anfgesetzten Tropftrichter und saugte sie erst dann in das Absorptionsgefäß hinein, wenn dieses bereits mit Stickoxyd gefüllt war. Auf diese Weise kann man sicher gehen, die Halogenwasserstoffsäure in der gleichen Stärke in Reaktion zu bringen, wie man sie bereitet hat. Nach diesem Verfahren haben wir auch zwei der früheren Versuche mit 11.94-n. Salzsäure wiederholt und das frühere Resultat, daß $1 \mathrm{NO}$ anf $1 \mathrm{Fe}$ gebunden wird, bestätigt gefunden (vergl. die Kurve in der früheren Abhandlung).

Der Bromwasserstoff wurde aus Brom und Naphthalin bereitet. Vor der Absorption durch Wasser wurde er, um Brom zurūckzuhalten, über roten Phosphor und durch ein mit einer Kältemischung gekühltes Rohr geleitet. Die so erhaltenen Bromwasserstoffsäuren waren ganz farblos, oder zeigten, wenn sie sebr konzentriert waren, in einigen Fällen doch nur eine Spur von Färbung. Fs soll jedoch nicht unerwähnt bleiben, daß beim Zusammentreffen des Stickoxydes mit den stärksten der benutzten BromwasserstoffLösungen letztere sich etwas gelb fărbten, eine Erscheinung, bei der schwer zu ermitteln ist, ob es sich um eine spurenweise Oxydation pon Bromwasserstoff durch Stickoxyd oder aber auch vielleicht un eine Verbindung zwischen Bromwasserstoff und Stickoxyd, ähnlich der früher von mir beschriebenen ron Sălzsäure und Stickoxyd ${ }^{1}$ ) handelt. Diese Nebenerscheinung ist jedoch

i) A. 375, 314 [1910]. 
zu unbedeutend, um as dem durch die Kurve wiedergegebenen Gesamtbild im Prinzip etwas zu ändern, immerhin könnte aber die Genavigkeit der mitgeteilten Zablen hierdorch etwas beeinträchtigt sein.

Die Stickoxydbindung im System $\mathrm{FeBr}_{2}+\mathrm{NO} \rightleftharpoons \mathrm{FeBr}_{2}, \mathrm{NO}$ wird, wie die Kurve zeigt, durch verdünnte Bronıwasserstoffsäure zunäichst, und zwar noch stärker als durch Salz- und Scbwefelsäure, zurückgedrängt. Mit zunebmeader Bromwasserstoff-Konzentration nimmt sie aber wieder zu und erreicht scbließlicb den Wert von 1 Molekiil Stickoxyd auf 1 Atom Eisen.

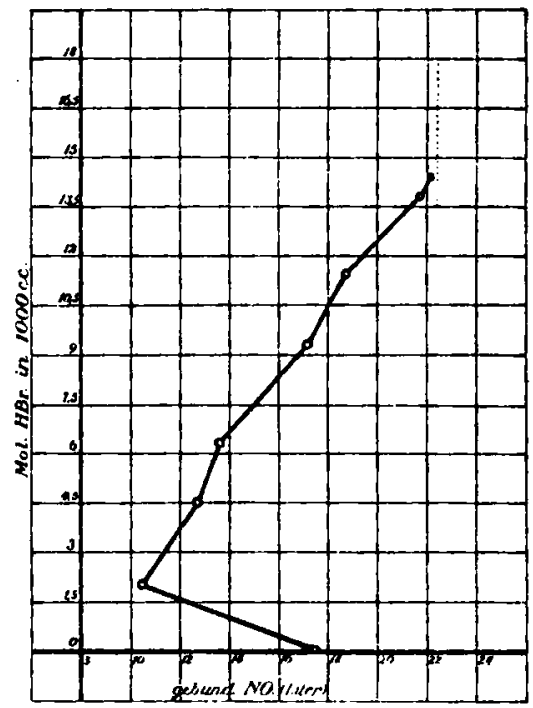

III. Kupferbromid-Stickoxyd, $\mathrm{CuBr}_{2}, \mathrm{NO}$.

In Fortsetzung meiner frïberen Versuche habe ich eine ausführliche Untersuchung darüber angestellt, ob die von mir quantitativ festgelegte Reaktion des Stickoxydes mit dem Kupfer-sulfat und -chlorid, $\mathrm{CuCl}_{2}+\mathrm{NO} \rightleftharpoons \mathrm{CuCl}_{2}, \mathrm{NO}$, eine allgemeine Reaktion der Kupiersalze ist. $Z u$ diesem $Z$ wecke wurde eine ganze Reihe von Kupfersalzen bei Gegenwart von absolutem Alkobol mit Stickoxyd in Reaktion gebracht. Geprüft wurden u. a. Kupfer-fluorid, -nitrat, - acetat, -tartrat, -benzoat, -formiat und das Kupfersalz des Glykokolls. Bei keinem dieser Salze konnte eine Bindung von Stickoxyd beobachtet werden. Ist dieses auch in den meisten Fällen durch die Unlöslichkeit der Kupfersalze in Alkohol und die Empfindlicbkeit der Stickoxyd-Verbindungen gegenüber der zersetzenden Wirkung des Wassers teilweise erklärlich, so bleibt es doch auffallend, daß in keinem Falle unter der Einwirkung des Stickoxydes das Kupfersalz in Iösung ging, indem etwa eine alkobollösliche Stickoxyd-Verbindung entstand. Bemerkenswert ist ferner, daß auch das in Alkohol lösliche Kupfernitrat kein Stickoxyd bindet und dieses negative Ergebnis nicht wohl durch die als Krystallwasser oder infolge der bygroskopischen Natur des Salzes eingebrachte Menge Wasser erklärt werden kann, da auch dann kein Stickoxyd gebunden wurde, wenn man der alkoholischen Lösung des Kupfernitrats entwässertes Natriumsulfat zusetzte. Bei einer alkoholischen Lösung von Kupieracetat und bei einer durch Kochen von Eisessig mit Kupfercarbonat bereiteten Lösung wurde zwar 
Gasabsorption beribachtet, diese ging jedoch wäbrend vieler Stunden inmmer weiter und schließlich über einen wahrscheinlichen Wert erheblich hinaus, ohne dali die Lösung die den Stickoxyd-Verbindungen charakteristische eigene intensive Farbe angenommen hätte. Die Färbung war vielmehr ichmutzigtrübe blaugrün und weit weniger intensiv. Es bandelt sich beer wabrscheinlich un Oxydationserscheinungen, jedenfalls um Nebenreaktiouen ganz anderer Art.

Auffallend bleibt namentlich das gänzlicbe Versagen der StickoxydBindung bei dem allerdings in Wasser und in Alkohol kaum löslichen Kupferfluorid, welches zu dem Verhalten von Chlorid und Bromid in eigentümlichem Giegensatz steht.

Unter diesen Umständen gewinnt die quantitative Untersuchung des bister nur qualitativ bekannten ") Additionsvermögeus vou $\mathrm{b}$ up $\mathrm{fer}$ bromid gegenuiber dem Stickoxyd ein viel gröljeres Interesse, als einem blolsen Analogiefall zukommen würde. Ich babe deshalb unter Nitwirkung meines Assistenten Dr. J. Has die nachstehenden Versuche ansgeführi.

\begin{tabular}{|c|c|c|c|c|c|c|c|}
\hline $\mathrm{Ne}$ & $\begin{array}{c}\text { Mol. } \\
\text { Cu Br: in } \\
1000 \text { ceed }\end{array}$ & $\begin{array}{l}\text { Gebund. } \\
\text { No anf } \\
\text { I CuBr: } \\
\text { (Litar) }\end{array}$ & $\begin{array}{l}\text { No- } \\
\text { Drnek } \\
\text { mn }\end{array}$ & $N$ is. & $\begin{array}{c}\text { Mol. } \\
\text { Cu Bra in } \\
1000 \mathrm{ccm}\end{array}$ & $\begin{array}{c}\text { Gobund. } \\
\mathrm{NO} \text { auf } \\
1 \mathrm{CuBr} \\
\text { (Liter) }\end{array}$ & $\begin{array}{c}\text { No- } \\
\text { Dluck } \\
\text { mm }\end{array}$ \\
\hline 1 & 0.00498 & 20.02 & 74.4 & 6 & 0.3833 & 20.90 & 749 \\
\hline 2 & 0.100548 & 20.32 & 749 & 7 & 0.1126 & 19.98 & 1119 \\
\hline 3 & 0.0107 & 21.36 & 746 & 8 & 0.05656 & 21.00 & 748 \\
\hline 4 & 0.0599 & 20.57 & 747 & 9 & 0.0504 & 18.66 & 334 \\
\hline 5 & $0.118 i$ & 21.48 & 747 & 10 & $\left.0.0478^{2}\right)$ & 19.07 & 746 \\
\hline
\end{tabular}

Versuchstemperatur bei Nr. 3 und $920.5^{\circ}$, sonst $0^{\circ}$.

Die Versuchsanordnung war die gleiche, wie früher beim Kupferchlorid. Unter NO-Druck ist der. Barometerstand bezw. der Barometerstand zuzinglich des Überdrucks bezw. abzüglich des Unterdrucks zu verstehen. Bei Versuch Nr. 9 mit Unterdruck ist ein mit Stickstoff verdünntes Stickoxyd angewendet; der Partialciruck wurde hier durch Analyse des über der Flüssigkeit stehenden Gases bestimmt. Die Tension des Alkohols ist bei dem angegebenen NODruck nicht abgezogen, aber bei der Berechnung des gebundenen NO-Volumens berücksichtigt.

Als Lösungsmittel diente ein durch Destillieren über gebranntem Kalk und Natriumäthylat möglichst sorgfältig entwässerter Alkobol bei Nr. 1-9, bei Nr. 10 gewöhnlicher Sprit. Die Resultate rou Nr. 1-6 dürfen als praktisch identisch angesehen werden. Die

1) Kohlschūtter, B. 37, 3044 [1904]; bezügl. d. Lit. vergl. A. 375, 308 [1910]. Die Erscheinung wurde zuerst am Kupfersulfat von Desbassins beobachtet.

I Sprit. 
Suhwankungen rübren jedenfalls nur daron her, daß der Wassergehalt des Alkohols bei den einzelnen Versuchen ein wenig verschieden ist, je nachdem, wie vollstïndig Entwässerung und Schutz vor Feuchtigkeit, was kaum zu kontrollieren, bis zum fertigen Füllen des Apparates gelungen sind. Gegen Wasser ist die NO-Verbinduug sehr empfindlich, und daher zeigt der mit wasserhaltigem 90-prozentigem Sprit ausgeführte Versuch Nr. 10 bereits eine deutliche Depression des gebundeneu Stickuxydes gegenüber den Versuchen mit wasserfreiem Alkohol. Die mit Pariationen von Druck und Temperatur ausgeführten Versuche zeigen entsprechend den irüher entwickelten Prinzipien, daß der Betrag von einem Molekül NO auf $1 \mathrm{CuBr}_{2}$ den Grenzwert der Stickoxyd-Bindung darstellt. Sie zeigen aber aich, dab alle diese Umstände bier etwas zuriicktreten vor dem überwiegenden Einfluß des Wassers. Ebeu deshalb sind auch die erbaltenen NO. Werte durchweg böber als die mit Kupferchlorid erhalteneu, weil das Kupferbronid im Gegensatz zun Chlorid kein Krystallwasser enthielt und in dem Zustand, in welchem es verwendet wurde, der Analyse zufolge nahezu 100-prozentig war (gef. 28.1, ber. f. $\mathrm{CuBr}: 28.3 \% \mathrm{Cu}$ ), also höchstens Spuren von Feuchtigkeit enthielt. Die Abbängigkeit de, Dissoziationsgrades vom Partialdruck wirł deutlicher als bei den ubigeu Versuchen, wenn man einen Wasserstofistrom durch die Reaktionsillüssigkeit bindurchschickt (vergl. die Denonstrationstersucle der folgeuden Abhaudlung), wodurch das Stickoxyd sehr rasch röllig ausgetritben wird. Die larbe der Lösungen ist ein tinten-artiges Blauschwarzvioleit.

Die Fähigkeit, NO zu addieren, ist also bcim Kupfer beschränkt auf Sulfat, Cblorid uud Bromid. Diese drei Kupfersalze verhalten sich wach meinen Versuchen in quantitativer Beriehung gleich. Sie binden alle drei in umkehrbarer Reaktion $\mathrm{CuR}_{2}+\mathrm{NO} \rightleftharpoons \mathrm{CuR}_{2}, \mathrm{NO}$ ein Molekül Stjckoxyd. Die enorme Gegenwirkung von Wasser gegen die Addition vọn NO macht es wabrscheiulich, daß hierbei die Anlagerung von $\mathrm{H}_{2} \mathrm{O}$ an das Molekül des Metallsalzes mit der Anlagerung von $\mathrm{NO}$ in Koukurrenz tritt.

\section{Versucbe über die Konstitution der Stickoxyd- Verbindungen.}

Ứberführungsversuche. Nie oben mitgeteilte Untersuchung über das feste Ferrostickoxyd-phosphat, $\mathrm{FeNO}, \mathrm{HPO}_{4}$, bestätigt in erfreulicher Weise die früher bei der Untersuchung der braunen wäßrigen Lösungen ron Manchot und Zechentmayer gewonnenen Resultate. Sie bestätigt zugleich meine früher über die Konstitution 
der braunen Ferrostickoxyd-Verbindungen geäußerte Ansicht ${ }^{1}$ ), nack welcher die braunen Verbindungen gewissermaßen als Analoga des Ferribydroxydes und der basischen Ferrisalze:<smiles></smiles><smiles>[14CH3][14C](O)(O)O</smiles>

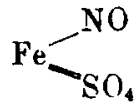<smiles>O=[Fe][Te]</smiles>

$\varkappa u$ betrachten sind und in den lösungen das Ion ( $\mathrm{FeNO}$ " ${ }^{*}$ enthalten. Diese Auffassung entspricht auch deu Beobachtungen bei Überführungsversuchen. Eine wäßrige 1/20-Ferroammoniumsulfat-Lösung wurde mit Stickoxyd gesättigt und die branne Flüssigkeit unter Benutzung der Nernstschen Versuclssanordnung mit ejuer äußerst verdünnten Schwefelsäure iberschichtet. Bei einer Spannung von 110 Volt und 0.1 Ampere stieg die braune Ferrostickoxyd-Lösung zur Katbode und fiel bei der Anode. Der Versuch wurde bäufig wiederbolt und natürlich stets durch Umkehrung der Stromrichtung kuntrolliert. Genau das Gleiche wurde beobachtet, als die grünbraune mit Stickoxyd gesättigte Lösung von $0.2 \mathrm{~g}$ Eisenchlorür in $100 \mathrm{ccm}$ Wasser mit äußerst verdünnter Salasäure in Überführungsapparat überschicbtet und auf das Verhalten beim Stromdurchgang gepriift wurde. Die brannen Lösungen enthalten also tatsächlich das Kation (FeNO) $"$.

Es dürte damit die Konstitution der braunen Stickwyd-Verbindungen sichergestellt sein.

Die grünen Ferrostickoxyd-Salze, von denen bisher noch keines außerhalb der Lösungen isoliert werden kounte, entbalten dagegen dem Überfübrungsversuch zafolge ein stickoxyd-haltiges Ferro-Anion. Die tiefgrüne Lösung, welche durch Sättigung von $0.2 \mathrm{~g}$ Eisenchlorür in $50 \mathrm{ccm}$ Alkobol mit Stickoxyd gewonuen wurde und durch Zusatz von $5 \mathrm{~g}$. krystallisiertem Chlorcalcium leitend gemacht war, wurde mit einer Lösung von $5 \mathrm{~g}$ krystallisiertem Cblorcalcium in 50 scm Sprit überschichtet. Beim Strumdurchgang hob sich die grüne Lösung zur Anode und fiel bei der Kathode.

Bezüglich der roten Lösung von Ferrosulfat-Stickoxyd in konzentrierter Schwefelsäure ergaben die Überlübrungstersucbe dagegen ein völlig negatives Resultat, wenngleich die Überschichtung der roten Jösung mit 90-prozentiger Schwefelsäure bier ganz besonders scharfe Girenzlinien von gefärbter und ungefärhter Schicht lieferte. Mit ganz schwachen Strömen verhielt sich die Lösung ganz indifferent, wäbrend bei größerer Stromstärke Verzerrungen der Grenzschicht eintraten, welche aber nur durch mechaniscbe Störuugen infolge des in der öligeu Schwefelsäure etwas erschwerten Entweichens der Gase an den

1) A. 37ะ, $153[1910]$. 
Elektroden veranlaßt waren und bald gegeuüber der Anode, bald gegenüber der Kathode auftraten.

Bezüglich der Kupferstickoxyd-Verbindungen ist, was zunächst die Lösung von Cuprisulfat-Stickoxyd in konzentrierter Schwefelsäure betrifft, zu sagen, daß sie sich ähnlich der roten Eisenverbindung ganz indifferent gegen den elektrischen Strom verhielt. Bei den Stickoxyd-Verbindungen von Cupri-chlorid und -bromid (in Alkobol) gaben die Versuche wegeu des geriugen Leitvermögens der Flüssigkeiten kein deutliches Bild, zumal Zusätze von Chlorcalcium, Harnstoff, Oxalsäure, etwas Wasser die Lösung rasch zersetzten.

Hervorzuheben ist hinsichtlich der Konstitution dieser Verbindungen, daß die dem braunen Typus der FerrostickoxydVerbindungen entsprechende Verbinduugsart beim Kupfer feblt ${ }^{2}$ ).

Zusammenhang zwischen Komplexbildung und Gasbindungsvermögen. Während das Bindungsvermögen für Stickoxyd eine charakteristische Eigenschaft der vormalen Ferroverbindungen $i_{\text {st, }}$ zeigen diejenigen Verbindungen des Eisenoxyduls, in welchen das Metall innerbalb eines sehr beständigen Sechserkomplexes gebunden ist, nicht die Fähigkeit, Stickoxyd zu binden. So bindet z. B. Ferrocyankalium in wäßriger Lösung kein Stickoxyd, und besonders charakteristisch ist in dieser Beziehung das Verbalten der Tri- $\alpha, \alpha-d$ ipyridylferrosalze $\left.{ }^{2}\right),\left(\mathrm{C}_{10} \mathrm{H}_{8} \mathrm{~N}_{2}\right)_{3}, \mathrm{Fe} \mathrm{R}_{2}$. Als ich zu einer mit Stickoxyd gesättigten Eisensulfat-Lösung eine zur Bildung der Tri-dipyridyl-Verbindung ausreichende Menge von $a, \alpha$-Dipyridyl ${ }^{3}$ ) gab, wurde das vorher vom Eisensulfat gebundene Stickoxyd quautitativ wieder abgespalten, während in der Lösung das intensiv rote Ferro-tri-dipyridyl-sulfat,

1) Damit wird auch die Meinung von Kohlschütter, B. 41, 1429 [1911] gegenstandslos, da $\beta$ meine Konstitutionsformel der braunen Eisenstickoxyd-Verbindungen konsequenterweise zur Annahme von dreiwertigem Kupfer führen würde . Koblschütter hat sich übrigens bereits mit den Überführungserscheinungen der Kupfer- sowie, nach meiner letzten Publikation, neuerdings auch der grünen nnd braunen Eisen-Lösungen beschäftigt. Aus dem soeben genannten Grunde und schon des Raumes wegen konnte ich auf seine komplizierte Betrachtungsweise jedoch nicht näher eingeben, zumal eine solche Diskussion das verhältnismåßig einfache Bild der Erscheinungen, welches sich aus meinen Beobachtungen ergibt, nur verwirren würde. Ich begnüge mich daher, meinen eigenen Befund in reproduzierbarer Form hier mitzuteilen.

2) F. Bla a, M. 19, 647 [1898].

3) Dargestellt nach Blau, M. 10, 372 [1889]; fiir die erforderliche $\mathrm{Pi}^{-}$ colinsăure wurde mit Vorteil die von C. Engler herrūhrende Modifikation des Weidelschen Verfahrens benutzt, vergl. Ar. 240, 345 [1902]. 
$\left(\mathrm{C}_{10} \mathrm{H}_{8} \mathrm{~N}_{2}\right)_{3}, \mathrm{FeSO} \mathrm{S}_{4}$, entstand. Entsprechend zeigte sich beim $\mathrm{Zt1-}$ sammenbringen vou Stickoxyd mit fertig hereitetem Dipyridylsalz, daß letzteres Stickoxyd uicht zu binden vermag.

Analoges habe jeh beziiglich des Additionsvermögens von Cuprisalzen gegen Stickoxyd beobachtet. Auch hier rermögen diejeoigen Verbindungen, welche einen beständigen komplexen Metallkern entbalteu, kein Stickoxyd zu binden. Eine mit stickoxyd gesättigte Lösung von Kupferbronid in Alkohnl spaltet nach meinen Beobachtungen anf Zusatz ron Dipyridyl das vorker gebundene Stickoxyd unter Entfärbung quantitativ wieder ab, indem sich Dipyridylcuprisalz ${ }^{1}, \mathrm{C}_{10} \mathrm{H}_{8} \mathrm{~N}_{2}, \mathrm{C}_{2} \mathrm{Br}_{2}$, ausscheidet, in welchem das Kupfer in komplexer Form gebunden ist. Dementsprechend ist dieses Salz, welches die normalen Kupfer-Keaktionen z. B. mit Natronlauge und nit Immoniak nicht gibt, in lertig bereiteten Zustand indifferent gegen Stickoxyd.

Ist dagegen eins der im Metallkern mit dem Eisenatom verbundenen 6 Radikale weniger fest gebundeu als die andren, wie es in den Salzen des ferro-pentacyan-ammins, $\mathrm{M}_{3} \mathrm{FeCy}_{5} \mathrm{R}$, der Fall ist ( $\mathrm{R}$ bedeutet $\mathrm{NH}_{3}$ bezw. Alkylamin), so ist das Additionsvermögen gegenwher Stickexyd uoch rorhanden $\left.{ }^{3}\right)$. Letzteres tritt dann unter Verdrängung des lockerer gebundenen Radikals $R$ in den Kern in fester Biadung ein, wobei, sei es uebenher, sei es als $Z_{\text {wischenprodutt, eine }}$ Verbindung entstebt, welche neben Stickoxyd nocb Alkylamin entbält, und wabrscheinlich eine Zwischenstufe bei der Verdrängung des Amins $R$ durch Stickoxyd rorstellt ${ }^{3}$ ).

Zusammenfassend möchte ich meine Ansicht von der Konstitution dieser Stickoxyd-Verbindungen so ausdrücken: die Fähigkeit. Stickoxyd zu addieren, ist eine charakteristische allgemeine Eigeuscbait aller normalen Ferroverbindungen, welche hierbei stets braune teil weise fest erbältliche Stickoxyd-Verbindungen bilden und, soweit letztere löslich sind, bei der Ionisation das Kation ( $\mathrm{FeNO}$ ) ' liefern. Die Eisen-Verbindungen nit komplexem Eisen-Anion dagegen addieren kein Stickoxyd, außer wenn wenigstens ein Bestandteil des Komplexes locker gebunden ist; dieser wird dann durch Stickoxyd verdrängt, $z . \mathrm{B}$. in $\mathrm{Na}_{3} \mathrm{FeCl}_{3} \mathrm{R}$. Daneben scheinen - vielleicht als

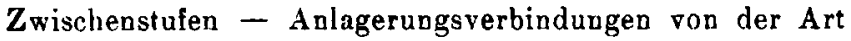

aufzutreten.

$$
\begin{gathered}
\mathrm{Na}_{8}\left[\mathrm{FeCy_{3 } \mathrm { Pyr }}\right] \\
\mathrm{NO}
\end{gathered}
$$

1) $\mathrm{Blau}$, a. a. 0.

Э) Manchot mit Merry und Woringer, B. 45, 2869 [1912].

3) Manchot und Woringer, B. 46, 3514 [1913]. 
Bei den komplexen Ferrosalzen erfolgt die Stickoxyd-Addition also nur, soweit durch die Komplexbildung die Gesamtaffinität des Moleküls noch nicht abgesättigt ist. Diese Stickoxyd-Verbindungen sind in elektrolytisch dissoziierenden Lösungsmitteln derart ionisiert, daß Eisen und Stickoxyd zusammen im Anion entbalten sind.

Die Gesamtheit aller Erscheinungen weist aber nach meiner Meinung darauf hin, daB die Anlagerung des Stickoxydes primär nicbt an das Ion, sondern an das ganze Molekül des Metallsalzes erfolgt, wobei letzteres zugleich aucb Salzsäure usw. addiert baben mag - und zwar sowohl in den braunen, roten und grünen Ferrostick oxyd-Lösungen wie in den Kupferlösungen. Denn alle Bedingungen, durch welche die Iouisation des Metallsalzes vermindert wird, verschieben zugleich das Gleicbgewicht

$$
\mathrm{MeX}_{2}+\mathrm{NO} \rightleftharpoons \mathrm{MeX}_{2}, \mathrm{NO}
$$

nach rechts, begünstigen also die Bindung von Stickoxyd'). Zum Beispiel lassen sich in grünen Lösungen zwar unter gewissen Bedingungen (s. oben) komplexe $\mathrm{FeNO}$-Anionen nachweisen, aber die grïne Farbe tritt gerade auch in organischen Lösungsmitteln von geringer oder gar keiner elektrolytischen Dissoziationskraft auf ${ }^{2}$ ), in manchen Fällen sogar unter besonders glatter und vollständiger Bindung eines Moleküls Stickoxyd. Die Entstehung stickoxyd-haltiger Metallionen - seien es Anionen oder Kationen - ist biernach eine sekundäre Erscheinung, also zunächst von mehr nebensächlicher Bedentung.

Bei den braunen Ferrostickoxyd-Verbindungen liegt wabrscheinlich ein Greuzfall vor, in welchem Anlagerung an das eisenhaltige Molek ül und Absättigung einer Hauptvalenz des Eisenatoms identische Vorgänge sind.

Zusammenhang zwischen Gasbindungsvermögen und roter Farbe. Meine oben mitgeteilte Beobachtung über die Tri- $x-$ dipyridyl-ferrosalze hat noch ein weiteres Interesse nämlich hinsichtlich der Frage, durch welche besondere Anordnung im Molekül der Eisenverbindungen das eigentümliche Additionsvermögen gegenüber Gasen zustande kommt, wie es der Blutfarbstoff besitzt. So hat wie schon früher F. Blau - neuerdings $W$ erner ${ }^{3}$ ) auf das spezielle

1) Eine scheinbare Ausnahme macht nur die Tatsache, daß konzentriertere Ferrolösungen weniger Stickoxyd aufnehmen als verdünnte. Hierbei macht sich aber eine andre Beeinflussung des Gleichgewichtes geltend, nämlich, daß dann die in der Volumeneinheit anf 1 Mol. Metallsalz kommende Menge Stickoxyd auch durch die verringerte Löslichkeit des Gases, also in doppelter Weise, vermindert wird.

2) Manchot und Huttner, a. a. O. 3) B. 45, 434 [1912]. 
Interesse hingewiesen, welches die Dipyridyl-ferrosalze verdienen, "weil sie durch ihre intensiv rote Farbe und die feste Bindung des Eisens an den Blutfarbstoff erinnern", ein Interesse, welches noch erböht worden ist durch die Versuche von Werner, denen zufolge diese Eisensalze in optisch-aktive Spiegelbildisomere spaltbar sind. Meine Beobachtung über das Verbalten der Tri.dipyridyl-ferrosalze gegen Stickoxyd zeigt nun, daß eigentümlicherweise diese roten Salze hinsichtlich der Annäberung an die wichtigste Eigenschaft des Blutfarbstofies, nämlich das Gasbindungsvermögen, weit zurückbleiben hinter vielen andren nicht oder scbwach gefärbten Ferrosalzen, unter denen wir nicht nur viele stickoxyd-addierende, sondern sogar kohlenoxyd ${ }^{1}$ )bindende jetzt kennen. Die rote Farbe ist also kein zuverlässiger Wegweiser für die künstliche Darstellung ron dem Blutiarbstoff im Gasbindungsvermögen ähnlichen Eisenverbindungen.

\section{W. Manchot: Demonstrationsversuche mit Ferro- stickoxyd-Verbindungen ${ }^{2}$ ).}

[Aus dem Chemischen Institut der Universität Würzburg.]

(Eingegangen am 7. Mai 1914.)

Die Reaktionen des Stickoxyds mit den Ferrosalzen ermöglichen einige leicht und glatt auszuführende Demonstrationsversuche, welcbe zugleich zur Erklürung des bekannten Salpetersäure-Nachweises dienen und zudem Gelegenbeit bieten, das Prinzip des chemischen Gleichgewichtes und die Erscheinungen einer umkehrbaren Reaktion zu erläutern. Man kann z. B. leicht zeigen, daß das Gleichgewicht $\mathrm{FeSO}_{4}+\mathrm{NO} \rightleftharpoons \mathrm{FeSO}_{4}, \mathrm{NO}$ durch Vermehrung der Eisen-Konzentration sich praktisch vollständig nach rechts verschieben läßt, indem man ein im "Nitrometer" abgesperrtes Volumen reinen Stickoxyds von einer kouzentrierten Ferrosulfat-Lösung vollständig absorbieren läßt. Andrerseits läßt sich die Umkehrbarkeit der Realtion in folgender Weise bequem demonstrieren. Die beiden Euden des Apparates rechts in der Figur sind das eine rnit einem Wasserstoff-Entwickler (Kipp), das andre mit einem Stickoxyd-(Gasometer ${ }^{3}$ ) verbunden. Die links aus-

1) Manchut, B. 45, 2869 [1912]; 46, 3514 [1913].

ग) Vergl. die voranstehende Athandlung.

3) Die für Yerbrennungen gebräuchlichen Gasometer mit über dem Gas. raum fest angebrachtem Wasser-heservoir sind für alle diese Versuche ganz ungeeignet. Besser benutzt man ein System von zwei großen, am Boden tubulierten und mit Schlauch verbundenen Flaschen. Farbloses Stickoxyd 